\title{
Necessidades de saúde comuns aos idosos: efetividade na oferta e utilização em atenção básica à saúde
}

\author{
Health care needs of the old aged: effectiveness \\ in the offer and use of primary health care services
}

Roberto Xavier Piccini 1

Luiz Augusto Facchini 1

Elaine Tomasi 2,3

Elaine Thumé 1

Denise Silva Silveira 1,3

Fernando Vinholes Siqueira 1,2

Maria Aparecida Rodrigues 1,3

\footnotetext{
1 Departamento de Medicina Social, Faculdade de Medicina. Av. Duque de Caxias 250, 96030-002, Pelotas RS. piccini@terra.com.br

2 Universidade Católica de Pelotas.

3 Secretaria Municipal da Saúde de Pelotas.
}

Abstract The effectiveness of the primary care offered and the use of these services made by the aged population approached in the baseline studies of the PROESF conducted by the UFPel included 41 municipalities of the States Alagoas, Paraíba, Pernambuco, Piauí and Rio Grande do Norte, Rio Grande do Sul and Santa Catarina. This transversal study was designed to characterize the structure and work process of 234 UBS, 4,749 workers and 4,003 aged people. The worst social indicators were observed in the Northeast of the country and in the PSF. There was a great need for regular domestic care and the prevalence rates of Systemic Arterial Hypertension (SAH) and Diabetes Mellitus (DM) were high. The use of protocols in domestic care was not very frequent and only half of the workers were capacitated to providing care to SAH and DM. Half of carriers of SAH and DM used the UBS of their region, practically all of them used medication, half of the individuals obtained their medication in the UBS and less than the half participated in the group activities in the UBS. A loss in effectiveness was observed in both in the offer and in the use of services. A comparison showed the performance of the PSF to be better that the traditional model. Key words Health of the aged, Family Health Program, Assessment of health services, Observational studies on public health interventions
Resumo A efetividade na oferta de serviços básicos e sua utilização por idosos abordada no Estudo de Linha de Base do Proesf conduzido pela UFPel incluiu 41 municípios dos Estados de Alagoas, Paraíba, Pernambuco, Piauí e Rio Grande do Norte, Rio Grande do Sul e Santa Catarina. O delineamento transversal caracterizou a estrutura e o processo de trabalho de 234 UBS, 4.749 trabalhadores e 4.003 idosos. Os indicadores sociais revelaram pior comportamento na região Nordeste e nas comunidades do Programa Saúde da Família (PSF). A necessidade de cuidados domiciliares regulares, a prevalência de Hipertensão Arterial Sistêmica (HAS) e de Diabetes Mellitus (DM) foram elevadas. Metade dos serviços oferecia barreiras arquitetônicas. O uso de protocolos para o cuidado domiciliar foi pouco freqüente e a capacitação para o cuidado de HAS e DM alcançou a metade dos trabalhadores. Metade dos portadores de HAS e DM usaram a Unidade Básica de Saúde (UBS) da área; praticamente todos usavam medicação, a metade obtinha a medicação na UBS e menos da metade participava das atividades de grupo na UBS. Perda de efetividade foi observada na oferta e na utilização de serviços. $O$ desempenho do PSF foi melhor quando comparado ao modelo tradicional.

Palavras-chave Saúde do idoso, Programa Saúde da Família, Avaliação de serviços de saúde, Estudos observacionais sobre intervenções em saúde pública 


\section{Introdução}

O SUS apresenta como porta de entrada no sistema a Atenção Básica à Saúde (ABS), nível em que aproximadamente $80 \%$ das necessidades de saúde de uma comunidade adstrita devem ser abordadas de maneira resolutiva. Neste nível, o Programa Saúde da Família (PSF) ocupou destacado papel nos últimos anos como estratégia indutora de eqüidade 1 .

O cuidado dos idosos implica ofertar serviços cuja estrutura apresente características que possibilitem o acesso e o acolhimento de maneira adequada, respeitando as limitações que proporções relevantes de idosos apresentam².

Os trabalhadores destes serviços devem estar capacitados em termos de conhecimentos, habilidades e atitudes para elaborar e operar protocolos para ações programáticas específicas às necessidades deste grupo populacional de maneira integrada com as demais práticas da rede de cuidado social ${ }^{3}$. Esta exigência se torna mais relevante quando se identifica a carência de especialistas e de serviços especializados neste núcleo do conhecimento à disposição do SUS4.

A avaliação da oferta e da utilização dos serviços de ABS por idosos permite medir a efetividade da política direcionada a este grupo populacional5.

O século que se inicia apresenta a perspectiva de revelar uma população idosa mais representada, tornando com isto a saúde dos idosos um dos grandes desafios para a saúde pública ${ }^{6}$.

Na região Nordeste do Brasil encontram-se cerca de $9 \%$ de idosos na população, sendo esta a menor proporção do País. A participação dos idosos na população alcança cerca de $10 \%$ na região Sul. A maior proporção de idosos é observada nos municípios de até 10 mil habitantes, diminuindo à medida que a população municipal cresce até alcançar o patamar de 300 mil habitantes, quando volta a crescer em função do aumento da população do município4.

O crescimento da população idosa brasileira tem provocado alterações profundas na sociedade. Este impacto, que deverá ser ainda maior no futuro ${ }^{7}$, é sentido na economia, no mercado de trabalho, nas relações familiares e no sistema de saúde.

$\mathrm{O}$ adequado enfrentamento das demandas causadas pelo envelhecimento da população significa reconhecer a projeção da Organização Mundial da Saúde de que até 2025 o Brasil será o sexto país do mundo em número de idosos e requer o conhecimento das características desta demanda 8,9 .

A Política Nacional do Idoso foi regulamentada pela lei no 8.842, de 4 de janeiro de 1994 - Cap. IV, art. 10, dispondo sobre os cuidados de saúde direcionados a este grupo populacional.

O Estatuto do Idoso, lei no 10.741 de 1o de outubro de 2003, prescreve diretrizes para o cuidado, com objetivos de prevenção e manutenção da saúde deste grupo populacional, incluindo ações de:

- Cadastramento da população idosa em base territorial.

- Atendimento domiciliar incluindo a internação para o idoso que precisar e estiver impossibilitado de se locomover, inclusive para aqueles abrigados e acolhidos por instituições públicas, filantrópicas ou sem fins lucrativos e eventualmente conveniadas com o Poder Público nos meios urbano e rural.

- Fornecimento gratuito, pelo Poder Público, de medicamentos, especialmente os de uso continuado, assim como próteses, órteses e outros recursos relativos ao tratamento, habilitação ou reabilitação.

As diretrizes e metas para a Reorganização da Atenção à Hipertensão Arterial Sistêmica (HAS) e a Diabetes Mellitus (DM) no Sistema Único de Saúde prevêem a atualização dos profissionais da rede básica, a garantia do diagnóstico e da vinculação do paciente às unidades de saúde para tratamento e acompanhamento, a promoção, reestruturação e ampliação do atendimento resolutivo e de qualidade para os portadores dessas patologias 10 .

As prescrições brasileiras revelam intenções para o cuidado integral dos idosos em sintonia com as experiências de países da União Européia. Na Itália, dentre os modelos inovadores, destaca-se o compartilhamento de informações entre os profissionais de diferentes setores. A padronização da comunicação, por meio de protocolos e formulários, está sendo desenvolvida na Dinamarca e na Alemanha. A incorporação de abordagem multidisciplinar é experimentada na Inglaterra e na Itália. A definição de um único serviço de referência para o cuidado de cada idoso, estabelecendo sua vinculação com quem promove o cuidado, é citada na Holanda e Inglaterra ${ }^{11}$.

A avaliação da implementação das políticas de saúde dirigidas aos idosos se reveste de significado especial para o direcionamento de recursos de maneira eficiente, eficaz e eqüânime ${ }^{12}$. 
O Estudo de Linha de Base (ELB) para o Monitoramento e Avaliação do Projeto de Expansão e Consolidação do Saúde da Família (Proesf), realizado pela Universidade Federal de Pelotas nas regiões Sul e Nordeste, obteve informações sobre a efetividade da oferta e utilização de serviços básicos de saúde em uma amostra populacional de idosos ${ }^{11}$.

Tomando como referência as prescrições da legislação e as recomendações para este segmento da população, o artigo apresenta análise em relação aos cuidados domiciliares e a portadores de necessidades crônicas como HAS e DM. $\mathrm{O}$ artigo também examina a plausibilidade de que o PSF determine uma maior efetividade na oferta e utilização destes cuidados do que o modelo tradicional (Figura 1).

\section{Metodologia}

O delineamento transversal do ELB utiliza grupos de comparação segundo o modelo de atenção básica à saúde e articula medidas de múltiplos níveis de agregação, as dimensões sob estudo (político-institucional, organizacional da atenção, cuidado integral e desempenho do sistema) e os níveis de análise empírica (gestão, unidades básicas de saúde e população) ${ }^{13}$. Para a comparação da efetividade dos modelos de atenção nas regiões estudadas, as UBS foram estratificadas em Tradicionais e PSF.

O universo do estudo foi constituído pela totalidade dos 41 municípios de mais de 100 mil habitantes, que compunham os Lotes 2 das regiões Sul e Nordeste, incluindo os Estados do Rio Grande do Sul e Santa Catarina, na região Sul, e Alagoas, Paraíba, Pernambuco, Piauí e Rio Grande do Norte, na região Nordeste ${ }^{14}$. Uma amostra estratificada por múltiplos estágios 15 , 16, 17 selecionou unidades básicas de saúde, profissionais de saúde, usuários e indivíduos residentes na área de abrangência dos serviços. A amostra de UBS foi proporcional ao tamanho da rede básica de saúde de cada município ${ }^{1}$.

Em cada UBS foram entrevistados todos os trabalhadores de saúde em atividade. Foram incluídos os profissionais de saúde de nível superior, os profissionais de nível médio e os Agentes Comunitários de Saúde. Considerando a falta de um denominador confiável, a amostra selecionada foi confrontada com o registro de profissionais lotados na UBS, obtido através do instrumento de avaliação da estrutura dos serviços.

A amostra populacional de idosos (pessoas com 65 anos ou mais) foi localizada na área de abrangência de cada uma das UBS. A estratégia para delimitação da área de abrangência incluiu a obtenção prévia do mapa da área de abrangência das UBS, uma estimativa populacional a partir das áreas censitárias do IBGE8. Estimouse uma amostra de 2.100 indivíduos em cada uma das regiões estudadas, cujo tamanho seria suficiente para examinar diferenças de $25 \%$ a $30 \%$ entre os modelos de atenção (PSF x Tradicional), com um poder estatístico de $80 \%$ e prevalências dos desfechos de, no mínimo, $25 \%$. Para a composição total da amostra foram esti-

\section{Figura 1}

Modelo teórico referencial para avaliar a efetividade do cuidado dos idosos.

Estudo de Linha de Base da ABS/ PSF. UFPel/ MS, Pelotas, 2006.
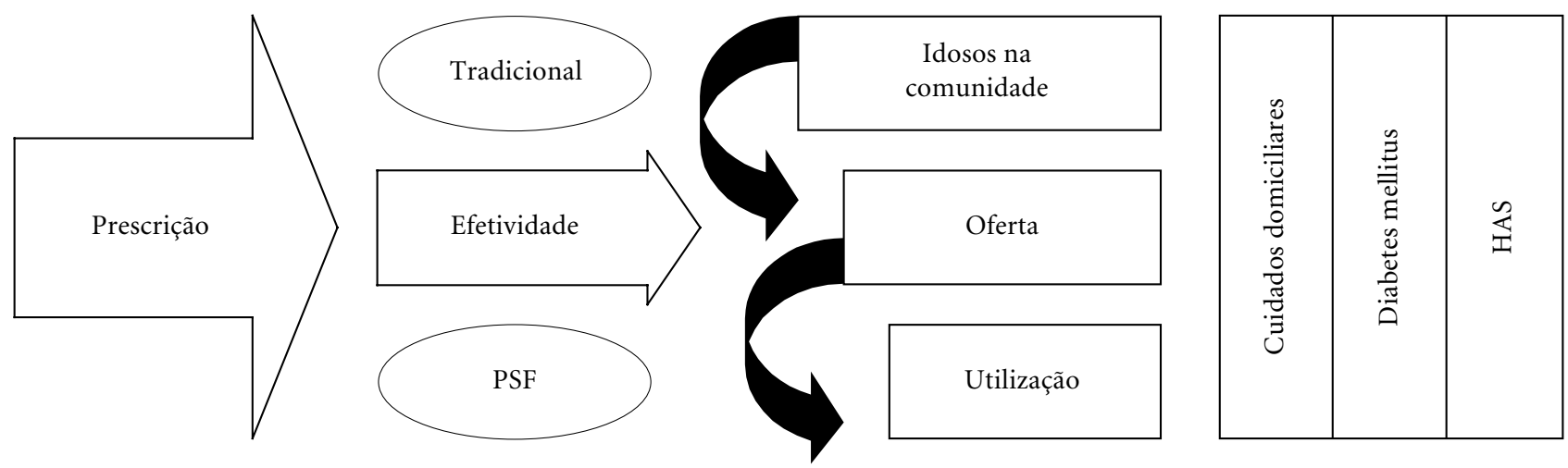
madas 18 entrevistas a serem realizadas na área de abrangência de cada UBS.

As atividades de campo, realizadas por uma equipe de 15 supervisores selecionados e capacitados para o seu desenvolvimento, duraram cinco meses (março a agosto de 2005).

$\mathrm{O}$ instrumento para captar informações sobre a estrutura das UBS era fechado, sendo preenchido coletivamente em uma reunião de equipe. Instrumento específico foi aplicado aos trabalhadores. Igualmente, através de questionário próprio, foram entrevistados os idosos em seus domicílios. A maioria das questões era estruturada e fechada, mas também havia questões abertas para qualificar as respostas fechadas.

A baixa densidade de idosos em cada domicílio facilitou a distribuição da amostra por toda a área de abrangência da UBS, minimizando as possibilidades de viés de seleção ou efeito de cluster nas amostras ${ }^{13}$. Nos domicílios selecionados, somente um idoso foi convidado a participar do estudo, explicando-se a sua finalidade e apresentando termo de consentimento informado. Nos domicílios em que se encontraram dois idosos, o elegível foi o mais velho.

Digitados no programa EPI-INFO 6.04b, os bancos de dados foram exportados através do aplicativo STAT TRANSFER 5.0 para o pacote estatístico SPSS 10.0 para Windows, utilizado para as análises. Inicialmente, procedeu-se às análises descritivas, verificando a distribuição dos casos em cada variável. Isto permitiu a validação da entrada de dados para amplitude e consistência das variáveis.

A análise bivariada examinou o comportamento das variáveis por dimensão teórica segundo o modelo de UBS (Tradicional, PSF). Na análise estratificada do efeito do modelo de UBS, os municípios foram agregados segundo a região (Sul e Nordeste). A estratificação possibilitou examinar as diferenças nos resultados por modelo de UBS em ambas as regiões.

Para variáveis dependentes do tipo qualitativas, dicotômicas ou nominais, as associações foram testadas por meio da comparação entre proporções, utilizando-se o teste do qui-quadrado. No caso de variáveis dependentes do tipo quantitativas, discretas ou contínuas, as associações foram testadas através da comparação entre médias, utilizando-se o teste F (ANOVA). O nível de confiança foi de $95 \%$, considerando significativas diferenças com $\mathrm{p}$-valor inferiores a 0,05 .

No exame da consistência dos dados de variáveis dicotômicas, utilizou-se o índice Kappa e, para as variáveis quantitativas, o coeficiente de correlação de Spearman ${ }^{18}$. Os índices de concordância para as questões refeitas em relação ao questionário dos idosos revelaram valores excelentes (acima de 0,7$)$ para a idade $(0,915)$, tabagismo $(0,822)$ e situação de aposentadoria $(0,783)$. Em níveis razoáveis $(0,35$ a 0,7$)$ estiveram a necessidade de tomar medicamentos para a hipertensão $(0,548)$ e para diabetes $(0,643)$, a necessidade de ajuda para percorrer uma quadra caminhando $(0,491)$ e a escolaridade do chefe da família $(0,550)$.

Os idosos caracterizados através de indicadores sociodemográficos, hábitos de vida, conhecimentos sobre hábitos saudáveis, percepção sobre a própria saúde e limitações na sua autonomia também foram investigados a respeito de necessidades de cuidados domiciliares regulares, prevalência de HAS e DM. Para esta demanda potencial foi avaliada a efetividade na oferta de serviços e em sua utilização, controlando-se o modelo de atenção (PSF e tradicional) e a região (Sul e Nordeste).

A análise de efetividade toma como parâmetros as prescrições do Sistema Único de Saúde para abordar este segmento da população e as necessidades são adotadas como marcadores neste estudo 3 . O desfecho estudado foi a necessidade de cuidados domiciliares regulares e as ações dirigidas a idosos portadores de HAS e DM. O primeiro nível da análise de efetividade se estabeleceu mediante a comparação da oferta de serviços para tais necessidades desta população de idosos com as prescrições das política. Um segundo nível desta avaliação identificou a proporção de utilização da oferta por aqueles idosos que portam estas necessidades ${ }^{13}$.

A oferta em atenção básica passa pela relação entre o número potencial de usuários adstritos e o dimensionamento deste serviço, a possibilidade de acesso, a adequação do serviço para acolher e resolver as necessidades demandadas e a capacitação dos trabalhadores para dar conta deste trabalho ${ }^{5}$.

\section{Resultados}

\section{Perfil sociodemográfico dos idosos na comunidade}

Os 4.003 idosos estudados nas regiões Sul (1.891) e Nordeste (2.112) revelaram, independentemente de modelo da atenção, semelhanças em relação à idade média (74 anos) e a gê- 
nero (60\% eram mulheres). Aproximadamente $40 \%$ eram casados(as) ou viviam com companheiro(a) e dois terços eram aposentados(as).

A predominância da cor da pele branca foi maior no Sul (83\%) do que no Nordeste (58\%). A informação de nenhuma escolaridade fez parte da história de $37 \%$ dos idosos no Sul (31\% na comunidade das UBS Tradicionais e $41 \%$ naquelas do PSF) e de $61 \%$ dos idosos do Nordeste (57\% na comunidade das UBS Tradicionais e $63 \%$ naquelas do PSF).

$\mathrm{O}$ analfabetismo foi a realidade encontrada em um quarto dos idosos do Sul, sendo $21 \%$ nas comunidades das UBS Tradicionais e $28 \%$ nas do PSF, enquanto no Nordeste era $41 \%$ a proporção de idosos analfabetos, 38\% nas comunidades das UBS Tradicionais e $42 \%$ nas do PSF.

A inclusão no grupo social menos privilegiado designado com "E” na classificação da Associação Brasileira de Empresas de Pesquisa (ABEP) - que é composta por informações sobre a escolaridade do chefe da família, a disponibilidade de empregada mensalista e sobre a posse de bens eletrodomésticos - foi verificada para 34\% dos idosos do Sul (30\% nas comunidades das unidades Tradicionais e 37\% nas do PSF) e para $56 \%$ do Nordeste ( $47 \%$ nas comunidades das unidades Tradicionais e 60\% nas do PSF). A renda média dos idosos do Sul $(1,1$ $\mathrm{SM})$ se mostrou superior em relação aos do Nordeste $(0,7$ SM) 19 .

Os domicílios foram considerados de construção adequada para 93\% dos idosos residentes nas áreas das UBS Tradicionais e para 91\% daqueles que residiam nas áreas das UBS do PSF, independentemente da região. Todos os domicílios tinham banheiro dentro de casa, mas a oferta de água encanada era maior no Sul (99\%) do que no Nordeste, onde as diferenças foram significativas entre os residentes nas áreas das UBS Tradicionais (93\%) do que nas áreas do PSF (90\%). O número de pessoas residentes por domicílio foi em média de 2,7 no Sul e de 3,8 no Nordeste, independentemente do modelo de atenção.

\section{Hábitos de vida dos idosos}

Aproximadamente metade dos idosos informou ter sido tabagista em algum momento de suas vidas, independentemente de região ou modelo de UBS.

Alimentar-se de forma saudável, não fumar, ingerir pequena quantidade de bebidas alcoólicas, realizar consultas regulares com o mé- dico e realizar exercícios físicos com regularidade foram conhecimentos considerados desejáveis para manter a boa saúde por dois terços dos idosos.

A informação de que havia caminhado por dez minutos ou mais na última semana esteve presente para $56 \%$ dos idosos do Sul e para $38 \%$ dos do Nordeste. O número médio de dias em que estes idosos caminharam dez minutos ou mais na última semana foi de 2,4 no Sul e 1,4 no Nordeste.

Atividades de média intensidade (provocava sudorese e aumentava os batimentos cardíacos) durante quatro ou mais dias na semana foram informadas por $44 \%$ dos idosos no Sul e $28 \%$, no Nordeste. Em ambas as regiões estes achados não revelaram diferenças em relação ao modelo de UBS ao qual pertenciam estes idosos.

\section{Necessidades de saúde dos idosos}

A população-alvo adstrita às UBS Tradicionais alcançou uma média estimada de 18.281 pessoas no Sul e 20.358 pessoas no Nordeste, enquanto a população adstrita para as unidades PSF mostrou uma média de 8.787 pessoas no Sul e 7.798 pessoas no Nordeste. É relevante destacar que no Nordeste aproximadamente 9\% da população corresponde a idosos, o que significa um número absoluto médio de 1.832 idosos por UBS Tradicional e 701 por unidade do PSF. No Sul esta proporção aproxima-se de $10 \%$, permitindo estimar a existência de 1.821 idosos por unidade Tradicional e 678 por unidade do PSF4.

A partir das proporções reveladas na caracterização da amostra do estudo, algumas das suas necessidades de saúde foram identificadas, especialmente aquelas crônicas e que demandam cuidados permanentes. Os números absolutos em cada célula constituem o denominador das proporções, ou seja, o número de idosos da amostra que responderam a cada um dos questionamentos tomados como variáveis neste recorte. (Tabela 1)

Um quinto dos idosos da região Sul (19\%) e um terço (28\%) do Nordeste não tinham autonomia para sair de suas casas sem companhia. Esta dificuldade, no Sul, foi significativamente maior entre os idosos das áreas do PSF $(22 \%)$ do que das UBS Tradicionais (15\%).

A necessidade de ajuda para caminhar uma quadra foi informada por $17 \%$ dos idosos da região Sul (15\% nas unidades Tradicionais e $18 \%$ nas unidades PSF) e $26 \%$ daqueles da re- 
Tabela 1

Necessidades de saúde dos idosos na área de abrangência das Unidades Básicas de Saúde. Estudo de Linha de Base da ABS/ PSF. PROESF, UFPel 2005.

\begin{tabular}{lcrcc}
\hline Característica & \multicolumn{2}{c}{ SUL } & & NE \\
& UBS Tradicional & UBS PSF & UBS Tradicional & UBS PSF \\
\hline Sem autonomia para sair de casa desacompanhado & $15 \%(793)$ & $22 \%(1.097)^{\star}$ & $29 \%(701)$ & $27 \%(1.406)$ \\
Necessidade de ajuda para caminhar uma quadra & $15 \%(793)$ & $18 \%(1.095)$ & $28 \%(699)$ & $24 \%(1.403)$ \\
Necessidade de auxílio p/ subir um lance de escada & $23 \%(794)$ & $28 \%(1.097)^{\star}$ & $37 \%(700)$ & $35 \%(1.403)$ \\
Necessidade de cuidados domiciliares regulares & $11 \%(784)$ & $19 \%(1.085)^{\star}$ & $24 \%(702)$ & $25 \%(1.404)$ \\
Portadores de hipertensão arterial sistêmica & $58 \%(791)$ & $64 \%(1.091)^{\star}$ & $64 \%(693)$ & $65 \%(1.388)$ \\
Portadores de diabetes mellitus & $18 \%(780)$ & $22 \%(1.083)^{\star}$ & $18 \%(681)$ & $19 \%(1.365)$ \\
\hline
\end{tabular}

${ }^{*} \mathrm{p}$ valor $<0,05$

gião Nordeste (28\% nas unidades Tradicionais $24 \%$ nas unidades PSF).

A necessidade de auxilio para subir um lance de escada foi informada por $26 \%$ dos idosos do Sul (23\% nas comunidades de UBS Tradicionais e $28 \%$ nas comunidades do PSF) e por $36 \%$ do Nordeste, neste caso sem diferenças significativas em relação aos modelos de UBS.

A necessidade de cuidados domiciliares regulares esteve presente para $11 \%$ dos idosos das comunidades das unidades Tradicionais e para $19 \%$ das do PSF na região Sul e para $24 \%$ dos idosos das comunidades das unidades Tradicionais e $25 \%$ das do PSF no Nordeste.

A prevalência de HAS alcançou quase dois terços dos idosos (62\% no Sul e $65 \%$ no Nordeste), enquanto a diabete mellitus atingiu um quinto (20\% no Sul e $19 \%$ no Nordeste). Na região Sul, 16\% da amostra de idosos referiu ser portador de hipertensão e diabete, sendo a diferença significativamente maior para os idosos residentes em áreas do PSF (18\%) do que nas áreas das UBS Tradicionais (13\%). No Nordeste esta proporção foi de $14 \%$ e não houve diferenças entre os modelos de atenção.

Quedas no último ano aconteceram para $35 \%$ dos idosos nas duas regiões, sendo $15 \%$ com fraturas no Sul e $9 \%$ no Nordeste, sem diferenças entre as populações dos dois modelos de UBS.

Um terço dos idosos percebia sua saúde como boa, muito boa ou excelente. Estes achados ocorreram independentemente de região ou modelo de UBS.

\section{Oferta dos serviços de ABS para os idosos}

A informação sobre a disponibilidade de equipes e trabalhadores foi obtida para 118 UBS em cada uma das regiões. Nas UBS estudadas havia em média 1,7 equipes, tanto no Sul quanto no Nordeste. Nas UBS do Sul havia em média 1,5 médicos; 1,6 enfermeiros; 2,7 auxiliares ou técnicos de enfermagem; e 6,7 ACS. Na região Nordeste, em cada uma das UBS, foi de 1,9 o número médio de médicos; de 1,9 o de enfermeiros; de 2,4 o de auxiliares ou técnicos de enfermagem; e de 12,0 o de ACS.

Foram identificadas nesta amostra de 236 UBS as características da oferta de serviços relacionadas à estrutura, equipamentos de apoio essenciais, capacitação de profissionais, adoção de protocolos para ações mais prevalentes e oferta de atividades com grupos. Os números absolutos apresentados entre parênteses constituem o denominador para cada uma das proporções identificadas para estas variáveis, como mostra a tabela 2 .

Na região Sul a metade das UBS Tradicionais foi considerada inadequada para pessoas com deficiência, sendo esta proporção significativamente menor do que a observada em UBS do PSF (68\%). Esta inadequação foi observada em 59\% das UBS do Nordeste, não havendo diferenças significativas entre os modelos de atenção (54\% Tradicionais e 63\% PSF).

A presença de degraus no acesso às UBS foi encontrada em praticamente metade das unidades ( $44 \%$ no Sul e $43 \%$ no Nordeste). No Sul, nas UBS do PSF, a existência de degraus no acesso ficou no limiar da significância estatística, sendo maior do que nas UBS Tradicionais, respectivamente $52 \%$ e $33 \%$. Dois terços (64\%) das unidades da região Sul e $58 \%$ das UBS do Nordeste não possuíam rampa de acesso.

Sala de espera sem cadeiras adequadas foi uma realidade encontrada em aproximadamente dois terços das UBS, alcançando $85 \%$ das uni- 
Tabela 2

Características da oferta de serviços na ABS. Estudo de Linha de Base da ABS/ PSF. Proesf, UFPel 2005.

\begin{tabular}{|c|c|c|c|c|}
\hline \multirow[t]{2}{*}{ Oferta de serviços } & \multicolumn{2}{|c|}{ SUL } & \multicolumn{2}{|c|}{ NE } \\
\hline & $\begin{array}{c}\text { UBS Tradicional } \\
\%(\mathrm{n})\end{array}$ & $\begin{array}{l}\text { UBS PSF } \\
\%(\mathrm{n})\end{array}$ & $\begin{array}{c}\text { UBS Tradicional } \\
\%(\mathrm{n})\end{array}$ & $\begin{array}{l}\text { UBS PSF } \\
\%(\mathrm{n})\end{array}$ \\
\hline \multicolumn{5}{|l|}{ Perfil da estrutura dos serviços } \\
\hline \multicolumn{5}{|l|}{ Adequação da estrutura } \\
\hline Inadequação do prédio $\mathrm{p} /$ pessoas c/ deficiências & $49 \%(46)$ & $68 \%(66)^{\star}$ & $54 \%(39)$ & $63 \%(78)$ \\
\hline UBS com degraus no acesso & $33 \%(48)$ & $52 \%(66)$ & $39 \%(39)$ & $47 \%(78)$ \\
\hline UBS sem rampa de acesso alternativa & $57 \%(47)$ & $71 \%(66)$ & $51 \%(39)$ & $65 \%(78)$ \\
\hline UBS sem cadeiras adequadas na sala de espera & $67 \%(49)$ & $63 \%(67)$ & $85 \%(39)$ & $64 \%(78)^{*}$ \\
\hline Ausência de cadeira de rodas na UBS & $59 \%(49)$ & $72 \%(67)$ & $72 \%(39)$ & $89 \%(78)^{*}$ \\
\hline \multicolumn{5}{|l|}{ Protocolos utilizados na UBS } \\
\hline Cuidados domiciliares & $23 \%(48)$ & $42 \%(65)^{*}$ & $19 \%(36)$ & $48 \%(73)^{*}$ \\
\hline Hipertensão arterial sistêmica & $71 \%(49)$ & $65 \%(65)$ & $71 \%(38)$ & $83 \%(76)$ \\
\hline Diabetes mellitus & $63 \%(49)$ & $65 \%(65)$ & $70 \%(37)$ & $81 \%(74)$ \\
\hline \multicolumn{5}{|l|}{ Realização de grupos com usuários } \\
\hline Grupo de hipertensos na UBS & $93 \%(29)$ & $95 \%(58)$ & $88 \%(24)$ & $96 \%(71)$ \\
\hline Grupo de diabéticos na UBS & $93 \%(29)$ & $93 \%(57)$ & $83 \%(24)$ & $94 \%(71)$ \\
\hline Grupo de idosos na UBS & $44 \%(23)$ & $65 \%(51)$ & $50 \%(24)$ & $81 \%(68)^{*}$ \\
\hline \multicolumn{5}{|l|}{ Perfil dos profissionais de saúde } \\
\hline Profissionais com capacitação p/ o cuidado da HAS & $44 \%(406)$ & $56 \%(787)^{\star}$ & $37 \%(922)$ & $50 \%(1190)^{*}$ \\
\hline Profissionais com capacitação p/ o cuidado da DM & $42 \%(406)$ & $56 \%(783)^{*}$ & $36 \%(910)$ & $48 \%(1174)^{*}$ \\
\hline
\end{tabular}

${ }^{*} \mathrm{p}$ valor $<0,05$

dades Tradicionais do Nordeste. A indisponibilidade de cadeira de rodas nas UBS foi identificada em dois terços das UBS, alcançando 89\% das unidades PSF do Nordeste.

O uso de protocolo para cuidados domiciliares foi detectado em $23 \%$ das unidades Tradicionais e em $45 \%$ das unidades PSF no Sul, enquanto no Nordeste 19\% das unidades Tradicionais e $52 \%$ das unidades PSF o utilizavam. A adoção de protocolos para ofertar os serviços de diagnóstico e tratamento de HAS e DM foi informada pela quase totalidade das UBS de ambos os lotes, não ocorrendo diferença entre modelos.

A oferta de atividades de grupo para HAS e DM foi informada por quase a totalidade das UBS no Sul, sem diferença entre os modelos. Na região Nordeste, a oferta nas UBS do PSF foi quase universal, caindo para $80 \%$ nas UBS Tradicionais. A oferta de atividades de grupo de idosos foi maior nas UBS do PSF tanto no Sul $(65 \%)$ quanto no Nordeste $(81 \%)$.

Os trabalhadores das UBS do PSF da região Sul revelaram diferenças significativas em relação a terem realizado capacitação para o atendimento de DM (56\%), em comparação com seus colegas das UBS Tradicionais (42\%). Em relação à hipertensão, as diferenças foram significativas, sendo de $56 \%$ no PSF e de $44 \%$ nas Tradicionais.

$\mathrm{Na}$ região Nordeste, as diferenças na capacitação dos trabalhadores para o cuidado de diabetes foram significativas, sendo de $36 \%$ nas UBS Tradicionais e $48 \%$ no PSF, enquanto a capacitação em hipertensão foi de $37 \%$ para os trabalhadores das unidades Tradicionais e de $50 \%$ para aqueles das unidades PSF.

\section{Utilização dos serviços de ABS por idosos}

A utilização dos serviços por idosos portadores de HAS e DM é apresentada na tabela 3. Os números absolutos constituem o denominador para as proporções apresentadas.

Menos da metade dos idosos diabéticos e hipertensos consultaram por este motivo nos serviços de ABS de sua área de abrangência, independentemente de região. Nas duas regiões a utilização dos serviços foi significativamente maior no PSF (cerca de 50\%) do que nas unidades Tradicionais (cerca de 35\%).

Aproximadamente dois terços dos idosos com HAS e DM agendaram sua consulta, 40\% 
Tabela 3

Utilização da UBS por idosos portadores de HAS e de DM. Estudo de Linha de Base da ABS/ PSF. Proesf, UFPel 2005.

\begin{tabular}{|c|c|c|c|c|}
\hline \multirow[t]{2}{*}{ Características da utilização de serviços } & \multicolumn{2}{|c|}{ SUL } & \multicolumn{2}{|c|}{ NE } \\
\hline & $\begin{array}{c}\text { UBS Tradicional } \\
\%(\mathrm{n})\end{array}$ & $\begin{array}{l}\text { UBS PSF } \\
\%(\mathrm{n})\end{array}$ & $\begin{array}{c}\text { UBS Tradicional } \\
\%(\mathrm{n})\end{array}$ & $\begin{array}{l}\text { UBS PSF } \\
\%(\mathrm{n})\end{array}$ \\
\hline \multicolumn{5}{|l|}{ Idosos com Hipertensão Arterial Sistêmica (HAS) } \\
\hline \multicolumn{5}{|l|}{ Perfil de utilização da UBS da área de abrangência } \\
\hline Consultaram na UBS & $37 \%(447)$ & $50 \%(663)^{\star}$ & $35 \%(435)$ & $53 \%(863)^{\star}$ \\
\hline Consulta agendada & $60 \%(163)$ & $68 \%(318)$ & $66 \%(142)$ & $66 \%(438)$ \\
\hline Espera superior a uma semana para consulta & $17 \%(162)$ & $16 \%(300)$ & $21 \%(143)$ & $32 \%(419)^{\star}$ \\
\hline Consultaram com o mesmo médico & $86 \%(161)$ & $76 \%(321)^{*}$ & $87 \%(150)$ & $92 \%(451)$ \\
\hline Utilizavam remédio para HAS & $96 \%(166)$ & $98 \%(330)$ & $94 \%(152)$ & $97 \%(454)$ \\
\hline Conseguiam medicação na UBS & $48 \%(153)$ & $53 \%(304)$ & $67 \%(131)$ & $76 \%(415)$ \\
\hline Participavam de grupo para HAS na UBS & $13 \%(166)$ & $31 \%(328)^{*}$ & $25 \%(152)$ & $39 \%(454)^{*}$ \\
\hline Hospitalização nos dois últimos anos por HAS & $14 \%(445)$ & $16 \%(678)$ & $18 \%(430)$ & $16 \%(898)$ \\
\hline \multicolumn{5}{|l|}{ Idosos com Diabetes Mellitus (DM) } \\
\hline \multicolumn{5}{|l|}{ Perfil de utilização da UBS da área de abrangência } \\
\hline Consultaram na UBS da área & $39 \%(134)$ & $53 \%(224)^{*}$ & $36 \%(117)$ & $48 \%(246)^{\star}$ \\
\hline Consulta agendada & $71 \%(51)$ & $73 \%(116)$ & $75 \%(40)$ & $64 \%(114)$ \\
\hline Espera superior a uma semana para consulta & $27 \%(52)$ & $15 \%(111)$ & $18 \%(39)$ & $36 \%(111)^{*}$ \\
\hline Consultaram com o mesmo médico & $89 \%(52)$ & $86 \%(118)$ & $93 \%(41)$ & $93 \%(118)$ \\
\hline Utilizavam remédio para DM & $85 \%(52)$ & $91 \%(116)$ & $86 \%(42)$ & $84 \%(118)$ \\
\hline Conseguiam medicação na UBS & $54 \%(50)$ & $59 \%(112)^{*}$ & $78 \%(37)$ & $71 \%(107)$ \\
\hline Participavam de grupo para DM na UBS & $10 \%(50)$ & $27 \%(118)^{\star}$ & $26 \%(42)$ & $40 \%(117)$ \\
\hline Hospitalização nos dois últimos anos por DM & $13 \%(135)$ & $15 \%(234)$ & $9 \%(116)$ & $13 \%(253)$ \\
\hline
\end{tabular}

* $\mathrm{p}$ valor $<0,05$

destes para o mesmo dia, sendo acolhidos a partir da fila. Cerca de um terço (Nordeste) a um quinto (Sul) destes idosos esperou mais de uma semana para se consultar, sendo esta proporção significativamente maior para portadores de HAS e DM das unidades PSF do Nordeste.

Consultar com o mesmo médico por HAS foi mais freqüente para os idosos das comunidades de UBS Tradicionais (quase 90\%) do que para aqueles das comunidades das UBS do PSF (76\%) no Sul. Consultar com o mesmo médico por DM foi observado para praticamente $90 \%$ dos idosos de ambos os lotes, sem diferenças em relação ao modelo de UBS.

O tempo médio decorrido desde a última consulta, tanto para HAS quanto para DM, foi de 45 dias nas duas regiões e para os dois modelos de UBS.

Praticamente todos os hipertensos (97\% no Sul e $88 \%$ no Nordeste) e a maioria dos diabéticos ( $88 \%$ no Sul e $85 \%$ no Nordeste) usavam medicamentos. As diferenças entre modelos não foram significativas.

Aproximadamente a metade dos hipertensos e diabéticos do Sul e dois terços do Nordeste conseguiram na UBS da área a medicação de uso contínuo informada em primeiro lugar entre o conjunto das medicações que usava.

A participação de idosos, na região Sul, em atividades de grupo dirigidas para HAS e DM foi de aproximadamente $10 \%$ nas unidades Tradicionais e $30 \%$ no PSF. Na região Nordeste, a participação de idosos em grupos para HAS e DM foi de 25\% nas unidades Tradicionais e 40\% no PSF.

Outras formas de tratamento que não aquelas preconizadas pelo médico foram procuradas por praticamente a metade dos idosos portadores de HAS e DM, independentemente de região ou modelo.

A hospitalização por HAS e DM alcançou aproximadamente $15 \%$ dos idosos em ambas as regiões, sem revelar diferenças entre os modelos de unidade.

\section{Discussão}

Na avaliação de intervenções em saúde pública, os estudos observacionais, conduzidos com rigor metodológico, são vantajosos em relação aos ensaios clínicos, embora todos os delineamentos 
epidemiológicos sejam passíveis de problemas e de viés $5,20,21,22,23$. A obtenção de dados primários individuais qualificou o estudo para além dos achados médios das análises de agregados, geralmente baseados em dados secundários e passíveis de viés ou falácia ecológica 22 .

A informação de não haver freqüentado a escola, o analfabetismo, a inclusão no grupo social menos privilegiado, a limitação na autonomia para sair de casa sem acompanhante, a necessidade de ajuda para caminhar uma quadra e para subir um único lance de escada alcançaram proporções quase duas vezes maior no Nordeste do que no Sul, revelando praticamente sempre um panorama pior nas comunidades do PSF em ambas as regiões. A estratégia do PSF parece ter se submetido rigorosamente ao princípio da eqüidade, tendo priorizado as comunidades menos privilegiadas quando de sua implantação.

Dois terços dos idosos revelaram conhecimentos considerados desejáveis para manter boa saúde, embora apenas um terço dos idosos tenha avaliado sua saúde positivamente, proporção inferior à encontrada em outro estudo de base populacional realizado no Sul do Brasil, onde a avaliação positiva alcançou 48,2\% dos entrevistados 2 . Esta diferença talvez se explique parcialmente devido ao fato de o estudo citado ter considerado idosas as pessoas com 60 anos de idade ou mais e o ELB considerar idosas pessoas com 65 anos e mais.

A recomendação médica de atividade física para os idosos foi pouco freqüente e a sua prática por idosos também. Esta recomendação revelou proporções muito semelhantes à encontrada para hipertensos de todas as idades (42\%) em um estudo transversal de base populacional realizado na cidade de Pelotas 24 .

Quedas e fraturas constituíram um evento freqüente neste grupo populacional, à semelhança da informação de outras fontes bibliográficas que registraram a ocorrência de uma queda por ano em um terço dos idosos que vivem em casa (MS/CAB no 4, 2000).

A prevalência de problemas crônico-degenerativos como HAS se mostrou mais elevada nesta amostra do que em outros estudos populacionais, nos quais a prevalência de HAS nesta categoria de idade era pouco maior do que $40 \% 2,24$. Há que se considerar o fato de as amostras dos estudos citados serem representativas da totalidade de um município, enquanto o ELB captou sua amostra da população adstrita às UBS, localizadas na periferia urbana, menos privilegiadas e com piores indicadores sociais, demográficos e de morbidade, sendo portanto previsível encontrar prevalências maiores deste problema crônico.

A demanda por cuidados domiciliares regulares se configurou como uma necessidade bastante freqüente entre os idosos da amostra, dado coerente com limitações já documentadas para os idosos em geral ${ }^{9} \mathrm{e}$, em especial, com as carências que os segmentos menos privilegiados da população revelam, enquanto a oferta desta modalidade de cuidado pelas UBS estudadas é extremamente reduzida, principalmente no modelo Tradicional.

A amostra populacional de idosos apontou uma realidade menos privilegiada nas comunidades do PSF em relação àquelas do modelo Tradicional, tanto na região Sul quanto na região Nordeste, embora nesta última a realidade tenha sido pior como um todo do que a da região Sul.

No PSF, o número estimado de pessoas adstritas às UBS em relação ao número de equipes e profissionais disponíveis foi aproximadamente duas vezes maior do que é preconizado 25 . Nas unidades Tradicionais esta situação foi mais crítica, considerando a falta de parâmetro para a definição de população adstrita. Este fato constitui um primeiro fator a contribuir para a perda de efetividade das políticas propostas para este nível de atenção, em especial para o cuidado dos idosos, que representam proporção significativa da demanda atendida nas UBS, além de exigirem um atendimento com dedicação de tempo um pouco maior devido à complexidade e cronicidade de seus problemas 9 .

A existência de barreiras arquitetônicas como degraus no acesso à UBS e a ausência de rampas foram observadas em uma significativa proporção dos serviços, o que significa a interposição de um verdadeiro muro entre o serviço e o usuário idoso, especialmente aqueles com limitações, que chegaram a representar mais de um terço da amostra estudada.

A realização de atividades educativas de grupo para idosos com HAS e DM foi uma prática ofertada por quase todas as UBS estudadas, sempre um pouco mais nas unidades PSF. Entretanto, a utilização destas atividades por idosos foi pequena, embora significativamente maior nas unidades do PSF.

A baixa prevalência de profissionais que tenham realizado capacitação para o cuidado de HAS e DM pode ter afetado a qualidade dos serviços ofertados e explicar sua baixa utiliza- 
ção pelos idosos, revelando uma contradição com a oferta quase universal destes cuidados nas UBS estudadas.

A desproporção entre a população a ser cuidada e o número de trabalhadores disponíveis, as absurdas barreiras arquitetônicas, a ausência de capacitação para as equipes, a pequena proporção de unidades com protocolos específicos e a baixa oferta de cuidados domiciliares representaram um primeiro conjunto de variáveis que contribuíram para a perda da efetividade do cuidado prescrito legalmente para este grupo populacional 25 .

Os dados permitem estimar que existe, em média, cerca de duas centenas de idosos em cada uma destas comunidades que permanecem afastados da possibilidade de receber cuidado. Impressiona o paradoxo entre a estimativa, em números absolutos, de idosos com necessidades de cuidados domiciliares regulares e a pequena proporção de unidades que possuía protocolo para este cuidado e a proporção ainda menor daquelas que ofereciam esta modalidade de cuidado. Esta situação foi mais acentuada nas unidades Tradicionais do que no PSF.

O cuidado dos idosos revelou uma perda seqüencial de efetividade, desde a oferta insuficiente em relação ao prescrito até a utilização que alcançou menos da metade da populaçãoalvo. Ainda assim, a demanda acolhida representou, em números absolutos, um elevado contingente para a média de trabalhadores identificada nas equipes de saúde. A efetividade também pode estar sendo comprometida pela qualidade do desempenho, sugerida através da elevada medicalização de idosos hipertensos e diabéticos, da baixa resolubilidade que pode ser presumida pelo fato de metade dos usuários buscar outras formas de tratamento além da preconizada pelo médico, além da elevada prevalência de hospitalização nos últimos dois anos por HAS, em relação aos padrões identificados na literatura 24 .

As unidades do tipo PSF se revelaram sistematicamente mais adequadas em relação ao prescrito do que as Tradicionais, embora ainda distante do adequado e desejável25. Portanto, o PSF, além de um mecanismo indutor da eqüidade no cuidado em saúde para os idosos, por alcançar mais aquelas populações que socialmente mais necessitam, se revelou também uma forma mais efetiva de promover o cuidado.

Os achados permitem concluir que novas iniciativas são necessárias para universalizar a cobertura do PSF, uma vez que sua efetividade foi maior do que a do modelo Tradicional. Considerando o fato de as unidades PSF estarem situadas em comunidades menos privilegiadas, talvez seja possível afirmar que o desempenho deste modelo seja ainda melhor do que o observado neste estudo. Um conjunto de recomendações pode ser feito para os formuladores de políticas a partir destas informações:

- O desenvolvimento, por motivos éticos, de políticas coerentes que superem a dicotomia entre os modelos, garantindo a toda a população de idosos o acesso aos cuidados do PSF.

- A eleição de objetivos realísticos para o cuidado integrado e integral do idoso, considerando suas necessidades de saúde e o investimento requerido para a capacitação de todos os profissionais, superando a ampla diferença cultural entre os provedores de cuidado social e de cuidado em saúde.

- A valorização das experiências inovadoras e a indução da iniciativa de compartilhar projetos de pesquisa em avaliação que incluam a capacitação dos atores implicados.

As recomendações descritas nos últimos três parágrafos se encontram em sintonia com aquelas preconizadas pela Comunidade Européia e registradas em fonte bibliográfica atual11. A estas recomendações poderiam se agregar outras tantas, cientificamente fundamentadas, para enfrentar o desafio da promoção de cuidados integrados, integrais e conseqüentes para os idosos na Atenção Básica à Saúde do Brasil. Entretanto, o complexo processo que se vislumbra necessário exige, parafraseando autor de língua portuguesa, respostas baseadas em "um direito que respeite e uma justiça que cumpra” 26 . 


\section{Colaboradores}

LA Facchini, RX Piccini, E Tomasi, E Thumé e Denise Silveira participaram da concepção, análise de dados, interpretação e redação final do artigo. FV Siqueira coordenou o trabalho de campo do estudo e participou da elaboração dos instrumentos e processamento de dados. MA Rodrigues participou do processamento de dados, elaboração dos instrumentos e revisão da literatura.

\section{Referências}

1. Facchini LA, Piccini RX, Tomasi E, Thumé E, Silveira DS. Projeto de Monitoramento e Avaliação do Programa de Expansão e Consolidação do Saúde da Família (Proesf): Relatório Final. Pelotas: UFPel; 2006.

2. Santos HB. O perfil de saúde dos idosos da região urbana de Pelotas e alguns de seus determinantes [dissertação]. Pelotas: Universidade Federal de Pelotas; 1999.

3. Brasil. Ministério da Saúde. Cad Atenção Básica no 4: Atenção à saúde do idoso. Brasília: MS; 2000.

4. Facchini LA, Piccini RX, Tomasi E. Subsídios à política de regulação do acesso, formação e capacitação de profissionais da saúde: perfil sociodemográfico, epidemiológico e capacidade instalada em saúde no Brasil. Relatório Final. Pelotas: UFPel; 2005.

5. Habicht JP, Victora CG, Vaughan JP. Evaluation designs for adequacy, plausibility and probability of public health programme performance and impact. Int J Epidemiol 1999;28(1):10-8.

6. Wilson C. The century ahead. Dædalus Winter 2006; 135(1).

7. Bós AMG, Bós AJG. Determinantes na escolha entre atendimento de saúde privada e pública por idosos. Rev Saúde Pub 2004;38(1):113-20.

8. IBGE (Instituto Brasileiro de Geografia e Estatística). Acesso e utilização de serviços de saúde: IBGE; 2003 [acessado em: jan. 2006] Disponível em: http:// www.ibge.gov.br/home/estatistica/populacao/trabalhoerendimento/pnad2003/saude/default.shtm

9. Silvestre JAN, Costa MM. Abordagem do idoso em programas de saúde da família. Cad. Saúde Pública 2003; 19(3):839-47.

10. Brasil. Ministério da Saúde. Secretaria de Ações Programáticas e Estratégicas. Plano de reorganização da atenção à hipertensão arterial e ao diabetes mellitus. Brasília: MS; 2001 [acessado em jan. 2006]. Disponível em: http://www.saude.mg.gov.br /ADO/Miolo2002PDF.pdf.

11. Lloyd J, Wait S. Integrated care: a guide for policymakers. Edinburgh; 2005 [acessado em jan. 2006]. Available from: http://www.ilcuk.org.uk/publications.cfm.

12. Vaughan R. Evaluation and public health. Am J Publ Health 2004;94(3):360.
13. Rothman K, Greenland S. Modern epidemiology. Philadelphia: Lippincott-Raven; 1998.

14. Brasil. Ministério da Saúde. Termo de referência para o estudo de linha de base nos municípios selecionados para o componente 1 do Proesf. Brasília: MS; 2004.

15. Lemeshow $S$, Hosmer D. Adequacy of sample size in health studies. Chichester: John Wiley; 1990.

16. Levy P, Lemeshow S. Sampling for health professionals. Belmont: LLP; 1980.

17. Lwanga S, Lemeshow S. Sample size determination in health studies: a practical manual. Geneva: WHO; 1991.

18. Landis JR, Koch GG. The measurement of observer agreement for categorical data. Biometrics 1977; 33(1):159-74

19. Associação Brasileira de Empresas de Pesquisa. Critério de classificação econômica Brasil. São Paulo: ABEP; 2003 [acessado em fev. 2006]. Disponível em: http://www.abep.org/codigosguias/ABEP_CCEB.pdf.

20. Black D, Morris J, Smith C, Townsend P. Inequalities in health: report of a Research Working Group. Washington: National Academy Press; 1993.

21. Des Jarlais DC, Lyles C, Crepaz N. Improving the reporting quality of nonrandomized evaluations of behavioral and public health interventions: the TREND statement. Am J Publ Health 2004; 94(3):361-6.

22. Heller RF, Page J. A population perspective to evidence based medicine: "evidence for population health". J Epidemiol Community Health 2002; 56(1):45-7.

23. Santos IS, Victora CG. Serviços de saúde: epidemiologia, pesquisa e avaliação. Cad Saúde Pública 2004; 20(Supl. 2):S337-41.

24. Piccini RX, Victora CG. Hipertensão arterial sistêmica em área no Sul do Brasil: prevalência e fatores de risco. Rev Saúde Pub 1994; 28(4):261-67.

25. Brasil. Ministério da Saúde. Guia Prático do Programa Saúde da Família. Brasília: MS; 2001

26. Saramago J. Prefácio. In: Salgado S, editor. Terra. São Paulo: Companhia das Letras; 1997.

Artigo apresentado em 27/02/2006

Aprovado em 31/03/2006

Versão final apresentada em 17/04/2006 